\title{
A new Technique for Solution of the Blasius and Falkner-Skan Boundary Layer Equations
}

\author{
A. Okasha El-Nady ${ }^{1}$, M. Fayek Abd Rabbo ${ }^{2}$ \\ ${ }^{I}$ Mechatronics Department, Faculty of Engineering /O6University, Egypt(Lecturer) \\ ${ }^{2}$ Mechanical Engineering Department, Shoubra faculty of Engineering /Benha University, Egypt
}

\begin{abstract}
The Blasius equation describing viscous flow over a flat plate has fascinated physicists, engineers, mathematicians and numerical analysts alike. This ODE is rich in physical, mathematical and numerical challenges. Because of its application to fluid flow, physicists and engineers have a keen interest in solving the Blasius equation and the related, but more general, Falkner-Skan $(F-S)$ equation. In the present paper, the Falkner-Skan (F-S) equation is solved using a new technique based on Taylor theory with shooting algorithm. The Falkner-Skan equation has two coefficients $\beta_{0}$ and $\beta$, which corresponding to different types of flows. The $3^{\text {rd }}$ order differential Falkner-Skan (F-S) equation is solved with different values of $\beta_{0}$ and $\beta$ using Matlab software. The results of the present technique are compared with the published results. Comparison shows an excellent agreement with the results that found in the literature.
\end{abstract}

Keywords: Falkner-Skan equation; Blasius flow; Nonlinear differential equation, Boundary layer

\section{Introduction}

Every since its first appearance in the literature in 1908 [1], the Blasius equation describing viscous flow over a flat plate has fascinated physicists, engineers, mathematicians and numerical analysts alike. This ODE is rich in physical, mathematical and numerical challenges. Two-dimensional flow over a fixed impenetrable surface creates a boundary layer as particles move more slowly near the surface than near the free stream. Because of its application to fluid flow, physicists and engineers have a keen interest in solving the Blasius equation and the related, but more general, Falkner-Skan (F-S) equation [2]. Since one can elegantly reduce these equations to one-dimensional non-linear ODEs through similarity arguments, mathematicians have found their fulfillment in uncovering the underlying symmetries and proving existence and (non-) uniqueness of its solutions. Unfortunately, a general analytical solution has not been forthcoming; however, for special cases of the F-S equation, several analytical solutions do exist. These prove most beneficial in verifying numerical algorithms. Numerical analysts, or as they are called by J.P Boyd, "arithmurgists" [3], have had a field-day with these equations. They offer the mystery of nonlinearity - yet the simplicity of one-dimensional - and the challenge of solving a boundary value problem through the determinism of an initial value problem. A host of numerical methods have emerged to solve these equations including, but not limited to, finite differences and finite elements. A list of nearly 150 references (some repeated) for these and other algorithms is found in Refs. 4 and 5.It is also noted that the majority of the numerical algorithms are based on Runge-Kutta ODE solvers, and therefore have a definite "black box" quality. In spite of the enormous numerical effort however, a truly simple, yet numerically accurate and robust algorithm is still missing. Many, if not all, algorithms to this point seem rather delicate in that their iterative strategies must be carefully tuned to avoid numerical instability. For example, most schemes require the initial guess of the shooting angle to be relatively close to the converged result, which does not make for a robust algorithm. Judging from recent literature, the general lack of numerical agreement to a consistent five or more digits, is indicative of the need for a reliable algorithm-- the development of which we now address. An approximate solution for solution of second order differential equation based on Taylor expansion is found in ref. 6. Our motivation in the present study is to obtain the solution of the Falkner-Skan (F-S)by a simple technique based on Taylor expansion. In this technique the third derivative Falkner-Skan (F-S)is obtained by direct substitution of the initial conditions in equilibrium differential equation. The function and its first and second derivatives at the next step are obtained using Taylor expansion. The third derivative of the next point is obtained from the equilibrium equation and so on. Results are obtained using Matlab software and compared with that published in the literature. Comparison shows an excellent agreement between the proposed technique and the published one.

\section{Basic Fundamentals}

In this section the basic fundamentals of Taylor's theorem as well as the forward, backward and central difference approximations of higher order derivative are reviewed.

Taylor's Theorem: If $f$ is a function continuous and $n$ times differentiable in an interval $[x, x+h]$, then there exists some point in this interval, denoted by $x+\lambda h$ for some $\lambda \in[0,1]$, such that 


$$
f(x+h)=f(x)+h f^{\prime}(x)+\frac{h^{2}}{2} f^{\prime \prime}(x)+\cdots \quad+\frac{h^{\mathrm{n}-1}}{(\mathrm{n}-1) !} f^{n-1}(x)+\frac{h^{\mathrm{n}}}{\mathrm{n} !} f^{n}(x+\lambda h)
$$

If $f$ is a so-called analytic function of which the derivatives of all orders exist,then one may consider increasing the value of $n$ indefinitely. Thus, if the condition holds that

$$
\lim _{n \rightarrow \infty} \frac{h^{n}}{n !} f^{n}(x)=0
$$

which is to say that the terms of the series converge to zero as their order increases, then an infinite-order Taylor-series expansion is available in the form of

$$
f(x+h)=\sum_{j=0}^{\infty} \frac{h^{\mathrm{j}}}{\mathrm{j} !} f^{j}(x)
$$

This is obtained simply by extending indefinitely the expression from Taylor's Theorem. In interpreting the summary notation for the expansion, one must be aware of the convention that $0 !=1$.

\section{Forward Difference:}

The first derivative of a function $f(x)$ can be approximated using forward difference:

$$
f^{\prime}(x)=\lim _{h \rightarrow 0} \frac{f(x+h)-f(x)}{h}+o(h)
$$

Backward Difference:

The first derivative of a function $f(x)$ can be approximated using backward difference:

$$
f^{\prime}(x)=\lim _{h \rightarrow 0} \frac{f(x)-f(x-h)}{h}+o(h)
$$

Central Difference:

The first derivative of a function $f(x)$ can be approximated using central difference:

$$
\begin{aligned}
f^{\prime}(x) & =\lim _{h \rightarrow 0} \frac{f(x+h)-f(x-h)}{h}-\frac{f^{\prime \prime \prime}(x)}{6} h^{2}+o\left(h^{3}\right) \\
f^{\prime \prime}(x) & =\lim _{h \rightarrow 0} \frac{f(x+h)-2 f(x)+f(x-h)}{h^{2}}+o\left(h^{2}\right)
\end{aligned}
$$

\section{The Problem of Falkner-Skan B.L}

The Falkner-Skan equation can be written in the nondimensional form as:

$$
f^{\prime \prime \prime}(\eta)+\beta_{0} f(\eta) f^{\prime \prime}(\eta)+\beta\left(1-f^{\prime}(\eta)^{2}\right]=0 ; \eta \in[0, \infty]
$$

With the following boundary conditions

$$
\begin{aligned}
& f(0)=0 \\
& f^{\prime}(0)=0 \\
& \lim _{\eta \rightarrow \infty} f^{\prime}(\eta)=1
\end{aligned}
$$

This equation can be solved as initial value problem using the shooting method. Through a specification of an additional initial condition to replace the condition at infinity, the boundary value problem transforms into an equivalent iterative initial value problem. In this case equation (7) is subjected to the following initial conditions:

$$
\begin{aligned}
& f(0)=0 \\
& f^{\prime}(0)=0 \\
& f^{\prime \prime}(0)=\alpha
\end{aligned}
$$

\subsection{Methodology of the Proposed Technique}

In this technique, the differential equation (7) is rearranged as follows:

$$
f^{\prime \prime \prime}(\eta)=-\beta_{0} f(\eta) f^{\prime \prime}(\eta)-\beta\left(1-\left(f^{\prime}(\eta)\right)^{2}\right)
$$

By direct substitution of the initial conditions given in (11), (12) and (13) the third derivative $f^{\prime \prime \prime}(0)$ atstarting

DOI: 10.9790/1684-1404014553 www.iosrjournals.org $\quad 46 \mid$ Page


point can be written as:

$$
f^{\prime \prime \prime}(0)=-\beta_{0} f(0) f^{\prime \prime}(0)-\beta\left(1-f^{\prime}(0)^{2}\right)
$$

The approximate function at $\eta+\Delta \eta$ is obtained using Taylor expansion (1) up to the fourth term:

$$
f(\eta+\Delta \eta)=f(\eta)+f^{\prime}(\eta) \Delta x+\frac{1}{2} f^{\prime \prime}(\eta) \Delta \eta^{2}+\frac{1}{6} f^{\prime \prime \prime}(\eta) \Delta \eta^{3}
$$

The first and second derivatives of the function $f(\eta)$ at $\eta+\Delta \eta$ are obtained using the central difference approximation (6-a) and (6-b) as:

$$
\begin{aligned}
& f^{\prime}(\eta+\Delta \eta)=\frac{f(\eta+2 \Delta \eta)-f(\eta)}{2 \Delta \eta}-\frac{f^{\prime \prime \prime}(\eta)}{6} \Delta \eta^{2} \\
& f^{\prime \prime}(\eta+\Delta \eta)=\frac{f(\eta+2 \Delta \eta)-2 f(\eta+\Delta \eta)+f(\eta)}{\Delta \eta^{2}}
\end{aligned}
$$

Then, the approximate third derivative at time $\eta+\Delta \eta$ is obtained using equation (14) as:

$$
f^{\prime \prime \prime}(\eta+\Delta \eta)=-\beta_{0} f(\eta+\Delta \eta) f^{\prime \prime}(\eta+\Delta \eta)-\beta\left(1-f^{\prime}(\eta+\Delta \eta)^{2}\right)
$$

So, the first iteration is obtained from equations (16) through (19) as:

$$
\begin{aligned}
& f(\Delta \eta)=f(0)+f^{\prime}(0) \Delta \eta+\frac{1}{2} f^{\prime \prime}(0) \Delta \eta^{2}+\frac{1}{6} f^{\prime \prime \prime}(0) \Delta \eta^{3} \\
& f(2 \Delta \eta)=f(0)+2 f^{\prime}(0) \Delta \eta+2 f^{\prime \prime}(0) \Delta \eta^{2}+\frac{4}{3} f^{\prime \prime \prime}(0) \Delta \eta^{3} \\
& f^{\prime}(\Delta \eta)=\frac{f(2 \Delta \eta)-f(0)}{2 \Delta \eta}-\frac{f^{\prime \prime \prime}(0)}{6} \Delta \eta^{2} \\
& f^{\prime \prime}(\Delta \eta)=\frac{f(2 \Delta \eta)-2 f(\Delta \eta)+f(0)}{\Delta \eta^{2}} \\
& f^{\prime \prime \prime}(\Delta \eta)=-\beta_{0} f(\Delta \eta) f^{\prime \prime}(\Delta \eta)-\beta\left(1-f^{\prime}(\Delta \eta)^{2}\right)
\end{aligned}
$$

The recurrence formula of this technique can be written as:

$$
\begin{aligned}
& f_{n}=f_{n-1}+f_{n-1}^{\prime} \Delta \eta+\frac{1}{2} f_{n-1}^{\prime \prime} \Delta \eta^{2}+\frac{1}{6} f_{n-1}^{\prime \prime \prime} \Delta \eta^{3} \\
& f_{n+1}=f_{n-1}+f_{n-1}^{\prime} \Delta \eta+2 f_{n-1}^{\prime \prime} \Delta \eta^{2}+\frac{4}{3} f_{n-1}^{\prime \prime \prime} \Delta \eta^{3} \\
& f_{n}^{\prime}=\frac{f_{n+1}-f_{n-1}}{2 \Delta \eta}+\frac{f_{n-1}^{\prime \prime \prime}}{6} \Delta \eta^{2} \\
& f_{n}^{\prime \prime}=\frac{f_{n+1}-2 f_{n}+f_{n-1}}{\Delta \eta^{2}} \\
& f_{n}^{\prime \prime \prime}=-\beta_{0} f_{n} f_{n}^{\prime \prime}-\beta\left(1-{f_{n}^{\prime}}^{2}\right)
\end{aligned}
$$

\subsection{Shooting Method}

One method for solving boundary-value problems - the shooting method - is based on converting the boundary-value problem into an equivalent initial-value problem. Generally, the equivalent system will not have sufficient initial conditions and so a guess is made for any undefined values. These guesses are changed until the final solution satisfies all the boundary conditions.

The shooting method is the preferred way to treat the F-S boundary value problem. The boundary condition $\lim _{\eta \rightarrow \infty} f^{\prime}(\eta)=1$ is replaced by the initial condition $f^{\prime \prime}(0)=\alpha$, where $\alpha$ is the skin friction coefficient. To be equivalent, the shooting angle $\alpha$ must be determined such thatlim $\operatorname{lom}_{\eta \rightarrow \infty} f^{\prime}(\eta)=1$. Except where noted for a particular range of $\beta$, the solution is assumed unique [7]. In this method it is most important to choose the appropriate finite values of $\eta \rightarrow \infty$. The solution process is repeated with another large value of $x \rightarrow \infty$ until two successive values of $f^{\prime \prime}(0)$ differ only after a desired digit signifying the limit of the boundary along $\eta$. The last value of $\eta \rightarrow \infty$ is chosen as appropriate value of the limit $\eta \rightarrow \infty$ for that particular set of parameters. Then the value of $f^{\prime \prime}(0)=\alpha$ is refined until the exact value of $\alpha$ is determined.

\section{Results and Discussion}

In the absence of an analytical solution of a problem, a numerical solution is indeed an obvious and a natural choice. Thus, the nonlinear third-order Falkner-Skan equation (7) with boundary conditions $(11,12,13)$, are solved using the present technique with shooting algorithm. The present technique was derived based on Taylor Expansion. To assess the validity and accuracy of the present method, comparison with previously reported data available in the literature has been made via Table I. 
Table I: Comparison of Values of $\mathrm{f}^{\prime \prime}(0)=\alpha$ For $\beta_{0}=1 / 2$ and $\beta=0$ From Different Authors

\begin{tabular}{|c|l|l|l|l|}
\hline \multicolumn{4}{|c|}{ values of $f^{\prime \prime}(0)=\alpha$} \\
\hline Asaithambi [7] & Zhang and Chen [8] & Saini et.al [10] & Ganapol[11] & Present \\
\hline 0.33205 & 0.33206 & 0.33243 & 0.332057 & 0.33200 \\
\hline
\end{tabular}

\subsection{Forward Flows}

The Falkner-Skan equation has two coefficients $\beta_{0}$ and $\beta$. The solutions corresponding to $\beta=0$ have being known as constant flows (Fig. 1), those corresponding to $\beta>0$ are called accelerating flows (Figs. $2-7$ ), and those corresponding to $\beta<0$ are known as decelerating flows. Physically relevant solutions exist only for $-0.1988<\beta \leqslant 2$. If $\beta_{0}=1 / 2$ and $\beta=0$, it is called Blasius flow (Fig. 1); if $\beta_{0}=1$ and $\beta=1 / 2$, it describes Homann axisymmetric stagnation flow (Fig. 2); if $\beta_{0}=1$ and $\beta=1$, it describes Hiemenz flow (Fig. 3 ); if $\beta_{0}=0$ and $\beta=1$, it is called Pohlhausen flow (Figs. 4-5); if $\beta_{0}=2$ and $\beta=1$, it represents the problem of Homann, describing the steady flow in the boundary layer along a surface of revolution near the stagnation point (Figs. 6-7). Sometimes, the Falkner-Skan equation specifically refers to $\beta_{0}=1$. Table II presents summary of Types of flow according to values of $\beta$ and $\beta_{0}$. The numerical results of $f^{\prime \prime}(0)$ corresponding to different $\beta \in[-0.1988, \infty]$ when $\beta_{0}=1$ are presented in Tables III and IV.

Table II: Types of Flow

\begin{tabular}{|c|c|l|}
\hline$\beta_{0}$ & $\beta$ & Type of Flow \\
\hline $1 / 2$ & 0 & Blasius flow \\
\hline 1 & $1 / 2$ & Homann axisymmetric stagnation flow \\
\hline 1 & 1 & Hiemenz flow \\
\hline 0 & 1 & Pohlhausen flow \\
\hline 2 & 1 & Homann steady flow in the boundary layer along a surface of revolution near the stagnation point \\
\hline 1 & $<0$ & Reverse flow \\
\hline
\end{tabular}

Table III: Comparison of Valuesoff ${ }^{\prime \prime}(0)=\alpha$ For $\beta_{0}=1$ and Different $\beta$ From Different Authors

\begin{tabular}{|c|l|l|l|c|}
\hline \multirow{2}{*}{$\begin{array}{c}\text { values } \\
\text { of } \beta\end{array}$} & \multicolumn{4}{|c|}{ values of $f^{\prime \prime}(0)=\alpha$} \\
\hline 40 & 7.314785 & Zhu et.al. [9] & Saini et.al. [10] & Present \\
\hline 30 & 6.338208 & 7.314785 & 7.318525 & 7.343106 \\
\hline 20 & 5.180718 & 6.338208 & 6.338051 & 6.359090 \\
\hline 15 & 4.491486 & 5.180718 & 5.180599 & 5.194342 \\
\hline 10 & 3.675234 & 4.491487 & 4.491677 & 4.501564 \\
\hline
\end{tabular}

Table IV: Comparison of Values of $f^{\prime \prime}(0)=\alpha$ For $\beta_{0}=1$ and Different $\beta$ From Different Authors

\begin{tabular}{|c|r|r|r|r|}
\hline \multirow{2}{*}{$\begin{array}{c}\text { values of } \\
\beta\end{array}$} & \multicolumn{4}{|c|}{ values of $\mathrm{f}^{\prime \prime}(0)=\alpha$} \\
\cline { 2 - 5 } & Zhang and Chen [8] & Zhu et.al. [9] & \multicolumn{1}{|c|}{ Saini et.al. [10] } & Present \\
\hline 2 & 1.687218 & 1.687218 & 1.687014 & 1.688429 \\
\hline 1 & 1.232587 & 1.232588 & 1.232465 & 1.233144 \\
\hline 0.5 & 0.927680 & 0.927680 & 0.927570 & 0.927900 \\
\hline 0 & 0.469600 & 0.469600 & 0.469593 & 0.469400 \\
\hline-0.1 & 0.319270 & 0.319270 & 0.319572 & 0.319000 \\
\hline-0.15 & 0.216362 & 0.216362 & 0.217352 & 0.216000 \\
\hline-0.18 & 0.128636 & 0.128637 & 0.129087 & 0.128100 \\
\hline-0.1988 & 0.005222 & 0.005225 & 0.006019 & 0.000000 \\
\hline
\end{tabular}

4.1.1.Blasius Flow $\left(\beta_{0}=1 / 2 \quad \beta=0\right)$

Applying the shooting algorithm, it is found that $\alpha$ lies between 0.35 and 0.3 . Refinement of $\alpha$ show that the exact value of $\alpha=0.3320$ 


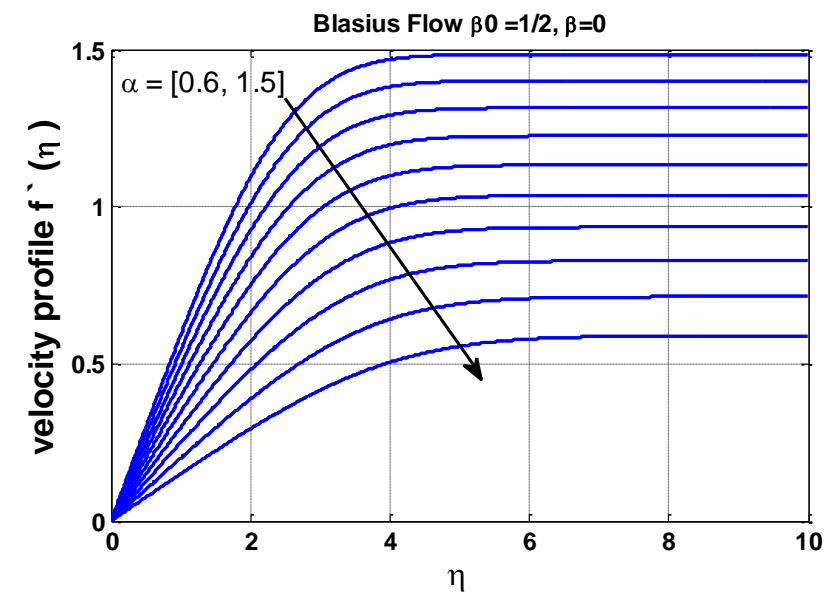

Fig. 1: Blasius Flow $\beta_{0}=1 / 2, \beta=0$

\subsubsection{Homann Axisymmetric Stagnation Flow $\left(\beta_{0}=1 \quad \beta=\frac{1}{2}\right)$}

Applying the shooting algorithm, it was found that $\alpha$ lies between 0.95 and 0.9 . Refinement of $\alpha$ show that the exact value of $\alpha=0.9279$

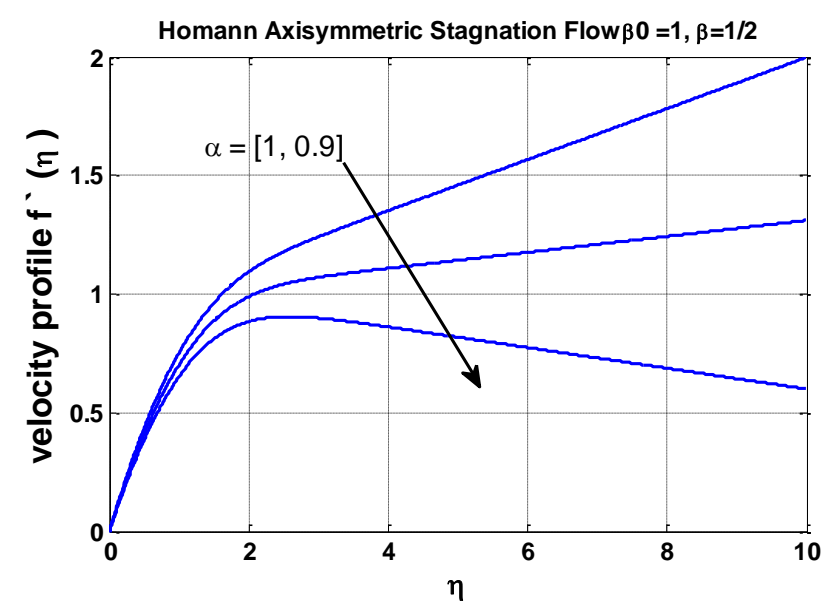

Fig. 2: Homann Axisymmetric Stagnation Flow $\beta_{0}=1 \quad \beta=\frac{1}{2}$

\subsubsection{Hiemenz Flow $\left(\beta_{0}=1 \quad \beta=1\right)$}

Applying the shooting algorithm, it is found that $\alpha$ lies between 1.25 and 1.2. Refinement of $\alpha$ show that the exact value of $\alpha=1.2331438$

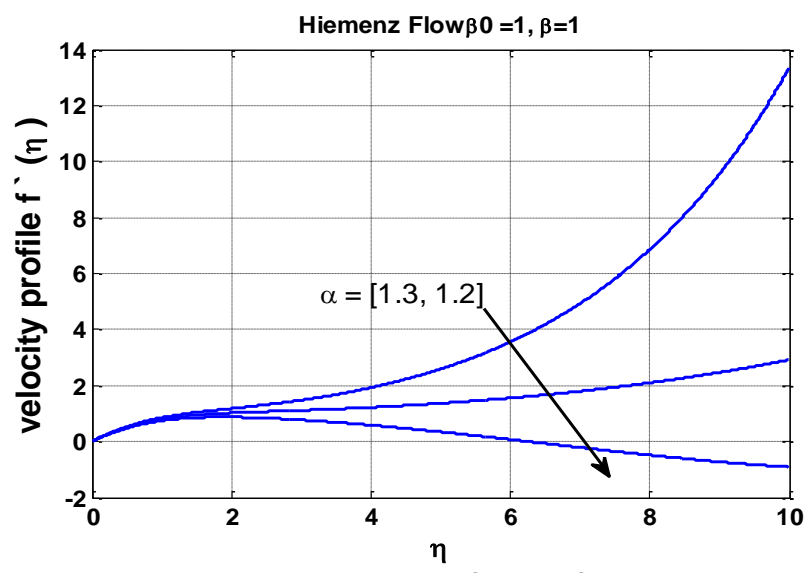

\subsubsection{Pohlhausen Flow $\left(\beta_{0}=0 \quad \beta=1\right)$}

Fig.3: Hiemenz Flow $\beta_{0}=1 \quad \beta=1$

Applying the shooting algorithm, it is found that $\alpha$ lies between 1.2 and 1.15. Refinement of $\alpha$ show that the exact value of $\alpha=1.15533595$ 
In this case the velocity profile is very sensitive to the value of $\alpha$ as shown in Fig. 5.

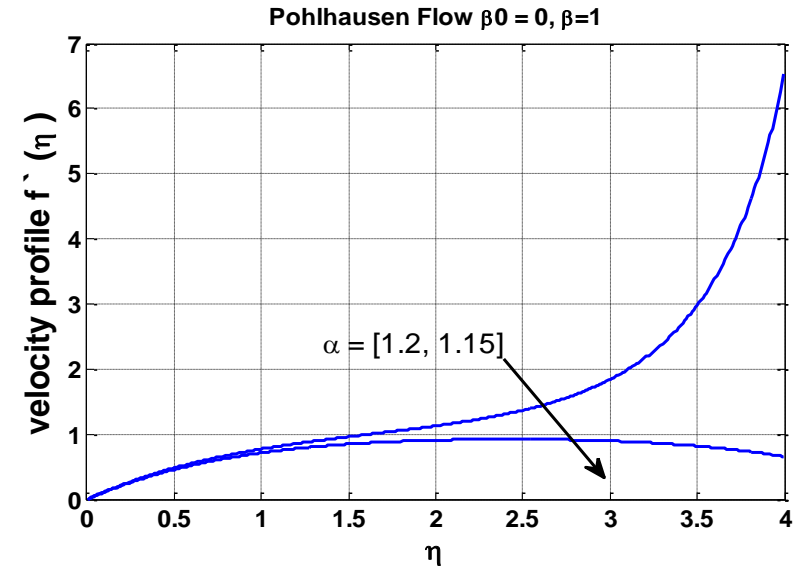

Fig.4: Pohlhausen Flow $\beta_{0}=0, \beta=1 \quad(\alpha=1.2: 1.15)$

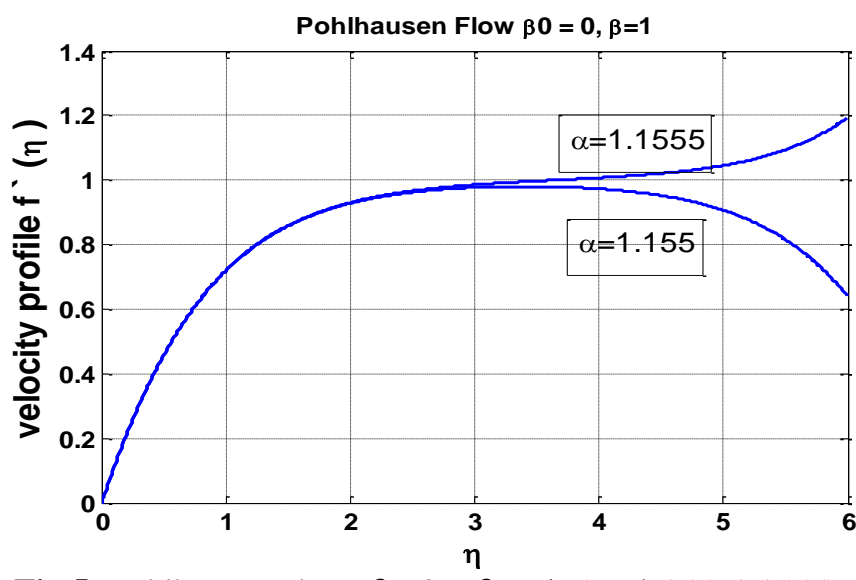

Fig.5: Pohlhausen Flow $\beta_{0}=0 \quad \beta=1 \quad(\alpha=1.155: 1.1555)$

\subsubsection{Homann Steady Flow $\left(\beta_{0}=2 \quad \beta=1\right)$}

Applying the shooting algorithm, it is found that $\alpha$ lies between 1.35 and 1.3. Refinement of $\alpha$ show that the exact value of $\alpha=1.31238$

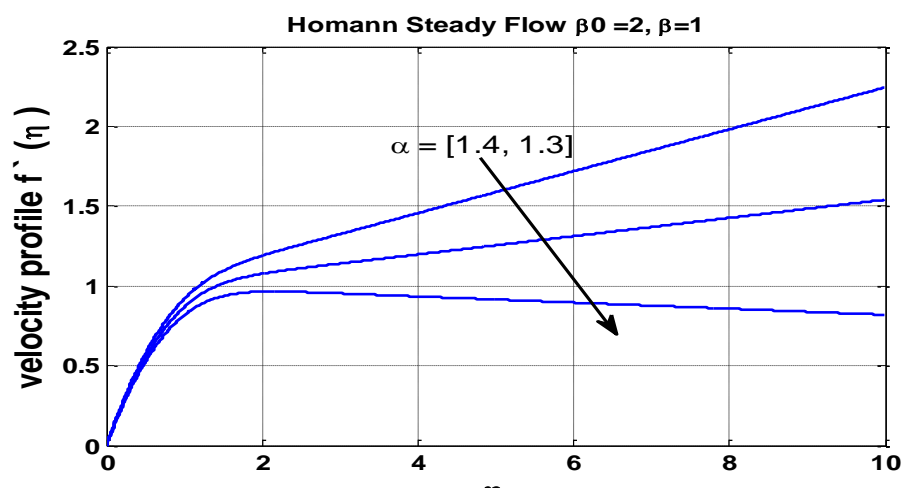

Fig.6: Homann SteadyFlow $\beta_{0}=2, \beta=1 \quad(\alpha=1.4: 1.3)$ 


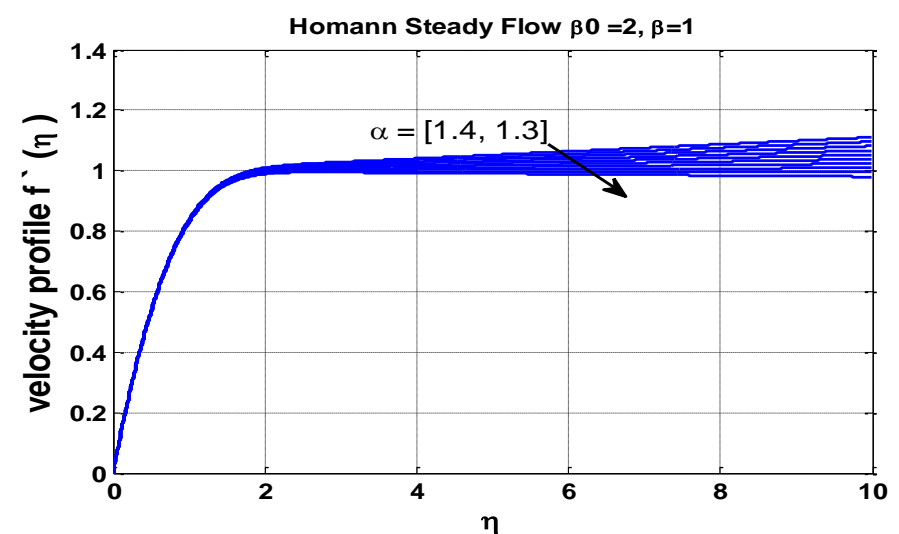

Fig. 7: Homann SteadyFlow $\beta_{0}=2 \quad \beta=1 \quad(\alpha=1.32: 1.31)$

\subsection{Reverse Flows}

A second solution of the F-S equation exists in the range $-0.1988<\beta \leqslant 0$ representing reverse flow. Figs. 8-10 show the variation of the velocity profile for a negative shooting angle for $\beta=-0.1$ and $\beta=-0.01$ with different values of $\alpha$ and compared with that in reference [10]. In each figure the upper part is the present and the lower part is taken from reference [10].
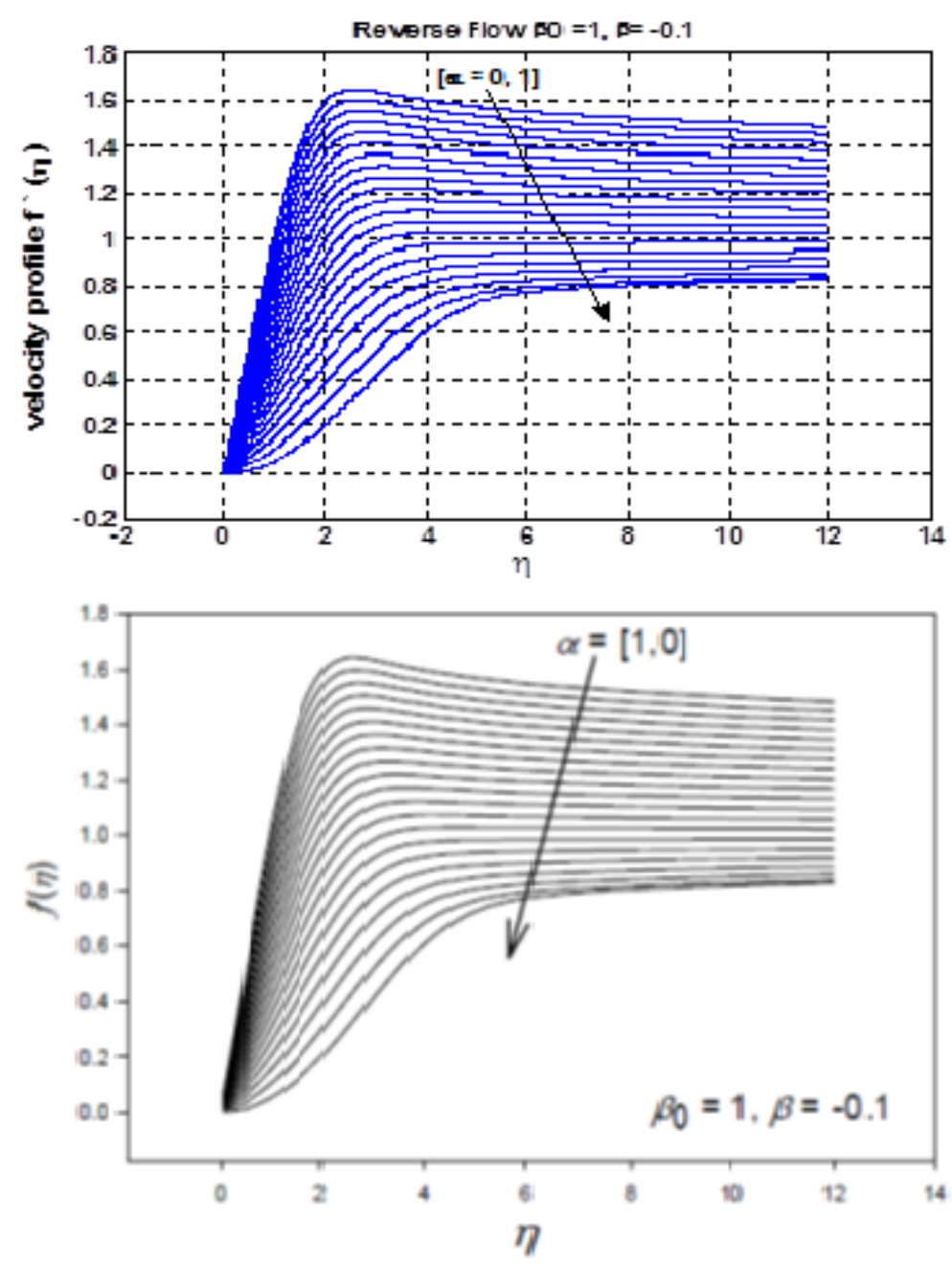

Fig. 8: Reverse Flow $\beta_{0}=1 \quad \beta=-0.1(\alpha>0)$ 

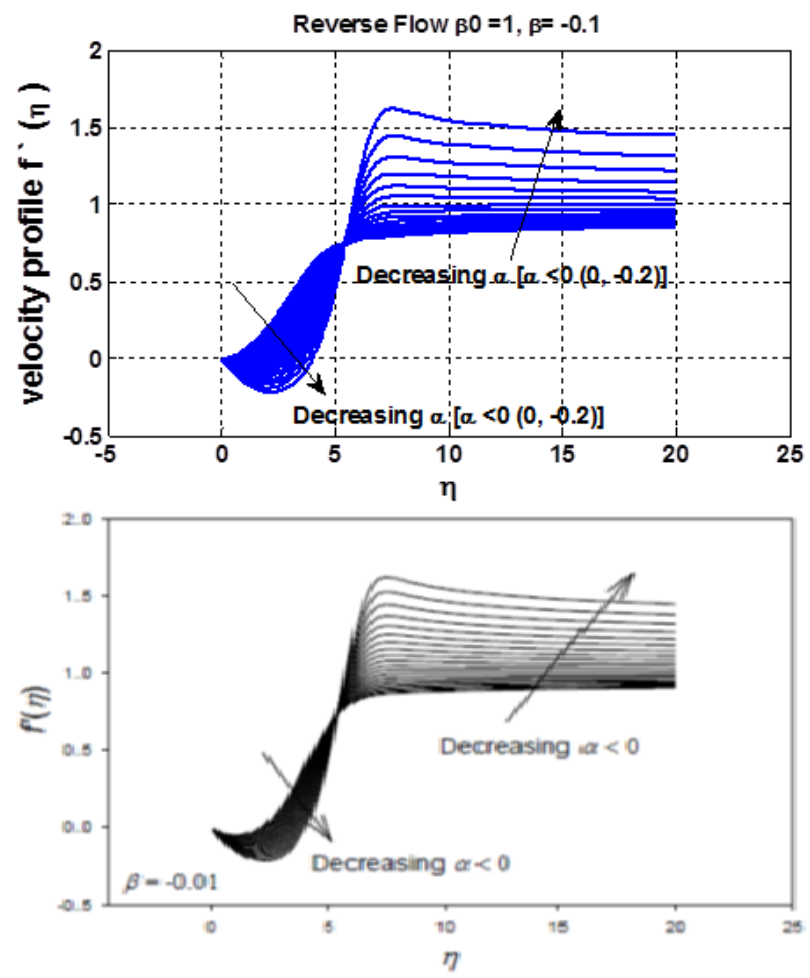

Fig.9: Reverse Flow $\beta_{0}=1, \beta=-0.1, \alpha<0(0:-0.2)$
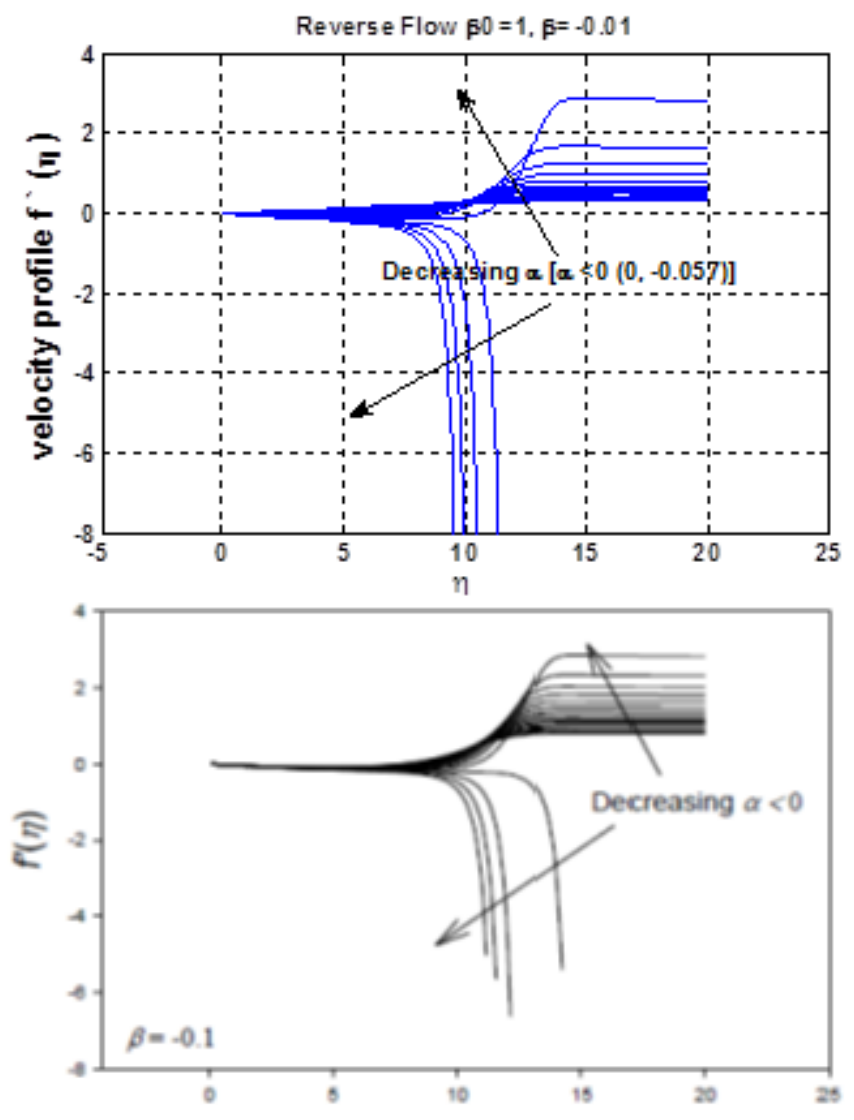

Fig. 10: Reverse Flow $\beta_{0}=1, \beta=-0.01[\alpha<0(0:-0.052)]$

\section{Conclusion}

In the present study, a new technique based on the Taylor expansion is applied to determine an approximate solution for the Falkner-Skan (F-S) third order differential equation. A comparison of the present 
results with the published results indicates excellent accuracy of the solution. It is concluded that the present technique is an accurate tool in handling the Falkner-Skan (F-S) equation with a high level of accuracy. Using the suggested technique, there is no need to transform the higher order differential equations to state space.

\section{References}

[1]. H. Blasius, Grenrschichten in FlussigkeitenmitkleinerReibung. Z. Math, u. Phys., 56, 1-37(1908).

[2]. V.M. Falkner, S.W. Skan, Some approximate solutions of the boundary layer equations, Philos. Mag. 12, 865-896 (1931).

[3]. J.P. Boyd, The BlasiusFunction : Computation Before Computers, the Value of Tricks, Undergraduate Projects, and Open Research Problems, SIAM Review, 50, 791-804(2008)

[4]. K. Parand a, M. Shahini a, Mehdi Dehghan, Solution of a laminar boundary layer flow via a numerical method, Comm..Nonlinear. Sci. Num. Sim. 15 360-367(2010).

[5]. URL Link: Steven Finch November 12, 2008 The boundary value problem y000 (x)+ ...

[6]. $\quad$ El-Nady, A. Okasha, and Maha MA Lashin. "Approximate Solution of Nonlinear Duffing Oscillator Using Taylor Expansion." Journal of Mechanical Engineering and Automation 6.5 (2016): 110-116.

[7]. A. Asaithambi, "A finite difference method for the solution of the Falkner-Skan equation", Applied Mathematics and Computation, vol.92, 1998, p.135-141.

[8]. J. Zhang and B.Chen, An iterative method for solving the Falkner-Skan equation, Applied Mathematics and Computation, 210, \#1, 215-222(2009).

[9]. S. Zhu, Q. Wu and X. Cheng, "Numerical solution of the Falkner-Skanequation based on quasilinearization", Applied Mathematics andComputation, vol. 215, 2009, p. 2472-2485.

[10]. SAINI, Indu; SINGH, Phool; MALIK, Vikas. "Genetic Algorithm Approach for Solving the Falkner-Skan Equation". Analysis, 2013, 10261: 9996966.

[11]. Ganapol, B. D. "Highly accurate solutions of the Blasius and Falkner-Skan boundary layer equations via convergence acceleration." arXiv preprint rXiv:1006.3888 (2010). 